\title{
Adverse reactions to nitrofurantoin in the United Kingdom, Sweden, and Holland
}

\author{
R G PENN，J P GRIFFIN
}

\begin{abstract}
A study was carried out comparing the reporting of adverse reactions to nitrofurantoin in Sweden, Holland, and the United Kingdom. The Swedish drug regulatory authorities receive more reports of adverse reactions to nitrofurantoin than to any other drug, and the annual reporting rate is increasing despite a steady fall in the prescription of nitrofurantoin. In the United Kingdom the total number of adverse reactions to nitrofurantoin reported to the Committee on Safety of Medicines has been steadily falling for many years, as has the number of prescriptions. Lung reactions and liver damage are more commonly reported in Sweden than in the UK, where gastrointestinal symptoms and peripheral neuropathy are more common. Such figures as were available in Holland showed an intermediate picture. The incidences of blood dyscrasias and allergic reactions are similar in the three countries.

The causes for such differences are unresolved, but it is disturbing that the incidence and range of adverse reactions to such a common drug as nitrofurantoin reported to the national authorities can differ so much in countries that have close ethnic, dietary, and economic similarities. Thus great caution must be observed when data on adverse drug reactions from one country are extrapolated to another.
\end{abstract}

\section{Introduction}

A recent paper ${ }^{1}$ analysed all reports of adverse reactions to nitrofurantoin sent to the Swedish Adverse Drug Reaction Committee from 1966 to 1976 . As urinary tract infection is the only approved indication for nitrofurantoin in both Sweden and the United Kingdom we compared the incidence and range of adverse reactions to nitrofurantoin in both countries.

\section{Methods}

Both Sweden and the UK have a national system for collecting and analysing data on adverse drug reactions. Information on reactions to nitrofurantoin in Sweden was primarily derived from the report by Holmberg et al. ${ }^{1}$ Some comparative data for Holland were also supplied by courtesy of the Netherlands Drug Registration Authority. The UK system is fundamentally voluntary, and the main input is from doctors reporting adverse reactions in their patients to the UK licensing authority on "yellow cards."

We used the original UK reports as the basis of our investigation so that the assessments could be presented in the same form as in the Swedish report. ${ }^{1}$ We scrutinised all cards mentioning nitrofurantoin either as the suspected drug or as concomitant treatment to eliminate cases in which nitrofurantoin was unlikely to be the cause of the reported reaction. Cards considered to report an adverse reaction probably or possibly due to nitrofurantoin were further subdivided

Department of Health and Social Security, London SW8 5NQ R G PENN, $M B, B C H$, principal medical officer

J P GRIFFIN, PHD, MRCP, senior principal medical officer using the categories and criteria described in the Swedish report ${ }^{1}$ and the following criteria.

Group 1: Acute pulmonary reactions-These reports described acute chest signs and were further subdivided into $(b)$ those in which bronchoconstriction was given as the major presenting complain and (a) the remainder ("pulmonary"). As in the Swedish study, this group was closely similar to the group showing allergic reactions except that the dominant symptoms were acute bronchoconstriction rather than cutaneous reactions.

Group 2: Chronic pulmonary reactions-Reports in this group were characterised by the duration of symptoms, which were often of chronic interstitial pneumonia.

Group 3: Allergic reactions-These included reactions ranging from rashes to anaphylaxis.

Group 4: Liver damage and gastrointestinal disturbances-These two symptoms were grouped together to facilitate comparison with the Swedish data, but we found it necessary to subdivide them into (a) liver damage, $(b)$ nausea and vomiting, and (c) various.

Group 5: Blood dyscrasias.

Group 6: Peripheral neuropathy.

Group 7: Various-A few reports did not fit the classifications for groups 1-6 in the Swedish scheme. We therefore created an additional category so that all reports could be included.

\section{Results}

Table I gives the absolute figures for adverse reactions to nitrofurantoin received by the drug regulatory authority in the UK over the years 1964-80. Nitrofurantoin was thought not to be causally related to the adverse reaction or to be dubiously so in 115 of the 570 yellow cards mentioning the drug. The remaining 455 reports were grouped as described above.

Table II shows the percentage of the total number of reports that each of these groups formed in Sweden (1966-76), the UK (1964-80), and Holland (1975-80). The percentage incidences of blood dyscrasias

TABLE I-Details of adverse reactions to nitrofurantoin in the UK (1964-80)

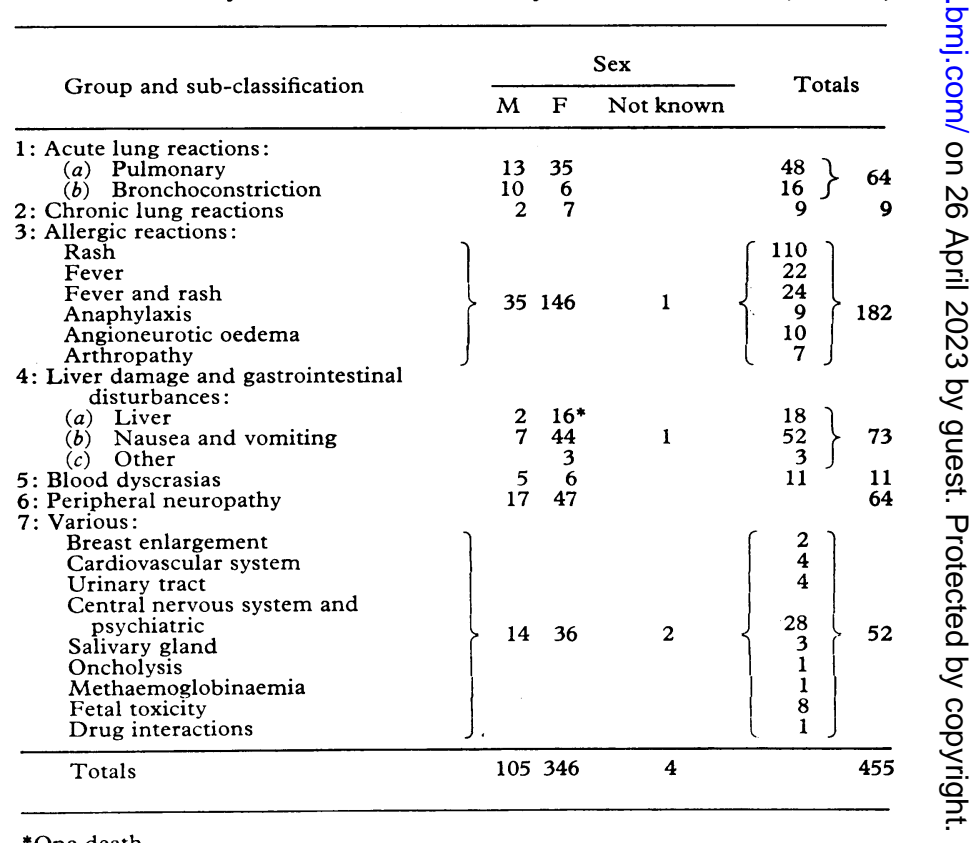

*One death. 
and allergic reactions were fairly similar between the countries, but there were considerable discrepancies in the other groups. Sweden had a greater number of absolute reports and a higher relative percentage of acute and chronic pulmonary reactions than either the UK or Holland. Sweden appeared to have a smaller total percentage $(5.4 \%)$ of reports of liver damage and gastrointestinal disturbances than the UK (16.0\%). Three-quarters of the UK reports, however, were of minor gastrointestinal upset such as nausea and vomiting with $3.9 \%$ being of potentially more serious liver disorders. The Swedish figure was more comparable with the latter UK figure, as Sweden had few reports of gastrointestinal disturbances. There was

TABLE II-Adverse reactions to nitrofurantoin as percentage of total reports received in the UK, Sweden, and Holland (absolute numbers in parentheses)

\begin{tabular}{|c|c|c|c|}
\hline Group & $\underset{(1964-80)^{*}}{\mathrm{UK}}$ & $\underset{(1966-76) \dagger}{\text { Sweden }}$ & $\underset{(1975-80) \ddagger}{\text { Holland }}$ \\
\hline $\begin{array}{l}\text { 1: Acute lung reactions } \\
\text { 2: Chronic lung reactions } \\
\text { 3: Allergic reactions } \\
\text { 4: (a) Liver damage } \\
\text { (b) Nausea and vomiting } \\
\text { (c) Various } \\
\text { 5: Blood dyscrasias } \\
\text { 6: Peripheral neuropathy } \\
\text { 7: Various }\end{array}$ & $\begin{aligned} & 14 \cdot 1(64) \\
& 2 \cdot 0(9) \\
& 40 \cdot 0(182) \\
& 3 \cdot 9 \\
& 11 \cdot 4 \\
& 0 \cdot 7 \\
& 2 \cdot 4 \\
& 14 \cdot 1 \\
& 11 \cdot 4(64) \\
& 11 \cdot 4(52)\end{aligned}$ & $\begin{array}{r}43 \cdot 2(398) \\
5 \cdot 3(49) \\
41 \cdot 7(384) \\
5 \cdot 4 \quad(50) \\
2 \cdot 2 \quad(20) \\
2 \cdot 2 \quad(20) \\
\text { Not known }\end{array}$ & 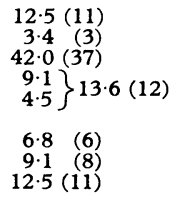 \\
\hline
\end{tabular}

* See Methods.

Tolmberg et al.

†Derived from data supplied by Netherlands Drug Registration Authority.

a higher incidence of neuropathy in the UK (14.1\%) than in Sweden $(2.2 \%)$; Holland occupied an intermediate position $(9.1 \%)$. Altogether $11.4 \%$ of the reports of reactions in the UK and $12.5 \%$ of the Dutch reports were not classifiable under the published Swedish scheme. The UK reactions were further subdivided (group 7; table I); they were a heterogenous group and unlikely to change the overall relative distribution of adverse reactions between the other groups. The 28 cases of central nervous system reactions included headache (four); dizziness, giddiness, and vertigo (seven); depression (four); confusion (three); hallucinations (one); schizophrenic reaction (one); slurred speech (two); drowsiness (two); abnormal vision (two) deafness (one); and shaking (one). Salivary gland enlargement occurred in three cases (all women) and was bilateral and painless; nitrofurantoin was the only drug given.

Twelve deaths were reported in the UK series, but in only one instance was nitrofurantoin thought likely to have been a contributory factor. In this case a woman aged 76 who had been taking nitrofurantoin for 18 months died of jaundice and hepatic failure but necropsy was not done. In the remaining 11 deaths it seemed reasonable to conclude that nitrofurantoin had had no appreciable role. Holmberg et al $^{1}$ listed 11 fatal reactions in which nitrofurantoin possibly had a role, although in five of these a second drug was considered to have been equally responsible. The remaining six fatal cases were considered to be due to a pulmonary reaction, either chronic (four) or acute (two)

\section{Discussion}

The present annual reporting rate of all adverse drug reactions is about $240 /$ million population in Sweden and 196/ million in the UK. In Sweden reporting of adverse reactions by doctors was voluntary between 1965 and 1974, but in 1975 reporting of fatal or otherwise severe reactions, as well as new or unexpected reactions, was made compulsory. Nevertheless, the reporting rate in the totally voluntary UK scheme is still $80 \%$ or more of that in Sweden.

In Sweden 921 reports of adverse reactions to nitrofurantoin were received in the years $1966-76,{ }^{1}$ which accounted for $8 \%$ of the total number of reports of adverse reactions to drugs for this period. In the UK the 455 reports received during the somewhat longer period 1964-80 represented only $0.5 \%$ of the total of 90000 reports received. This difference is even more pronounced when these figures are related to the population of the two countries, giving 10.5 reports/million inhabitants/year for Sweden and 0.5 reports/million inhabitants/year for the UK.
Urinary tract infection is the approved indication for nitrofurantoin in Sweden, Holland, and the UK. It is, of course, impossible to say whether in practice use of nitrofurantoin in Sweden and the UK follows the recommended dosage $(50 \mathrm{mg}$ three or four times daily for up to two to three weeks in Sweden; $100 \mathrm{mg}$ four times daily for two weeks in the UK). There has been a fashion in the treatment of urinary tract infection to give various drugs including nitrofurantoin as long-term follow-up, particularly when dealing with chronic pyelonephritis in women. Indeed, in the one case in the UK in which death was attributed to nitrofurantoin the drug had been given for 18 months.

In the UK the proportion of adverse reactions to nitrofurantoin to the total number of adverse reactions reported for all drugs fell steadily from $1.77 \%$ in 1964 to $0.11 \%$ in 1980 . In Sweden, however, the reverse applied. Nitrofurantoin provided $1.5 \%$ (46) of the total number of adverse reactions in 1965-9, $7.9 \%(526)$ in $1970-4$, and $7 \cdot 2 \%(742)$ in $1975-9$; during this period the ranking of nitrofurantoin as a cause of adverse reactions rose from tenth to second, and by 1980 it was first (B E Wiholm, personal communication). Thus not only is there a difference between the numbers of reports of adverse reactions to nitrofurantoin received by the drug regulatory authorities of Sweden, Holland, and the UK but the range of the reactions is different.

We should have liked to obtain figures of drug usage in each country for each year for the past 15 years so that we could assess whether these differences were merely artefacts of usage. It is not easy to compare quantitatively drug usage between countries as the methods of gathering data and the units used are often different. In the UK, for example, prescribing statistics are given in terms of general practitioner prescribing for the NHS whereas in Sweden they are given in terms of the number of defined daily doses issued (the defined daily dose for nitrofurantoin is $200 \mathrm{mg}$ ). It is obvious, however, even with these disparate units, that prescription of nitrofurantoin is falling in both countries. In the UK prescriptions of nitrofurantoin fell from 265000 in 1976 to 208200 in 1979, a fall of $21 \%$. In Sweden the fall was from 4543000 defined daily doses in 1976 to 2779000 in 1979, a fall of $39 \%$.

The difference in reporting rates in terms of the proportions of the total number of reports of adverse reactions was 16-fold ( $8 \%$ for Sweden $v 0.5 \%$ for the UK) and per head of population was of the same order-namely, 21 -fold $(10.5$ reports/million inhabitants for Sweden and 0.5 for the UK). The difference in reporting rates per 1000 prescriptions, however, has been estimated to be about 10 -fold (0.9-1.2 reports/prescription in Sweden and 0.07-0.08 in the UK). An analysis of rates of prescribing of nitrofurantoin in Sweden and the UK (B E Wiholm, personal communication) indicates that for the years $1976-9$ the rate was $23 \cdot 6-15 \cdot 3$ prescriptions/1000 inhabitants in Sweden and 4.7-3.7 in the UK.

That adverse reactions to a particular drug vary from country to country is, of course, not a new finding. Iodinated 8-hydroxyquinolines such as clioquinol have, for example, been associated with the development of subacute myelo-optic neuropathy in Japan, but the occurrence elsewhere is much disputed as is the importance of ethnic and dietary factors. Nevertheless, it is disturbing to find that the range of side effects to such a common drug as nitrofurantoin can differ so much between countries like Sweden, Holland, and UK, with their obvious close ethnic, dietary, and economic similarities. This paper poses two questions: to what extent do ranges of adverse reactions differ from country to country with other drugs; and do any such differences depend on the actual incidence of adverse reactions or on the importance placed on them by the doctor reporting them to the drug regulatory authorities ? It is as well to issue a cautionary note, therefore, that direct comparisons of the ranges of adverse reactions to any drug cannot be made between countries, even those that at first sight seem similar.

Factors that may influence the reporting rate are the activities of the drug regulatory authority in issuing warnings about the 
adverse reactions to a particular drug. Both the Swedish and UK regulatory agencies issued warnings, but their substance and emphasis differed, and this may have introduced some bias; neither regulatory authority, though, emphasised the problem of peripheral neuropathy, which appears to be the major difference in the profiles of adverse reactions to the drug.

We are grateful to Dr B E Wiholm of the National Board of Health and Welfare, Department of Drugs, Sweden, and Dr B H Ch Stricker of the Netherlands Centre for the Monitoring of Adverse Reactions to Drugs for their help and for supplying national data. We are also grateful to the Committee on Safety of Medicines for permission to quote from the UK Adverse Reactions Register, and to Professor Abe Goldberg for his invaluable help and criticism.

\section{Reference}

${ }^{1}$ Holmberg L, Boman G, Bottiger LE, Eriksson B, Spross R, Wessling A. Adverse reactions to nitrofurantoin. $A m \mathcal{F}$ Med 1980;69:733-8.

(Accepted 23 March 1982)

\section{SHORT REPORTS}

\section{Unusual complication of perforated appendix}

Peritonitis, local or generalised, is a common accompaniment of acute appendicitis, and residual septic foci may occur. The incidence of these has decreased over recent years, partly as a result of improved surgical techniques but largely owing to the use of prophylactic antimicrobial agents, ${ }^{1}$ whether local ${ }^{23}$ or systemic. ${ }^{4}$

We routinely use antibiotic peritoneal lavage (using a solution of $1 \mathrm{~g}$ tetracycline $/ 10.9 \%$ saline) in cases of peritoneal sepsis, with good results. ${ }^{2}{ }^{3}$ Residual intraperitoneal sepsis may still occasionally occur in the form of pelvic, interloop, and subphrenic abscesses, in addition to local sepsis in relation to the appendix stump. We describe an abscess in the scrotum after appendicectomy.

\section{Case report}

A 9-year-old boy was admitted with a two-day history of colicky central abdominal pain that localised in the right iliac fossa on the day of admission. $\mathrm{He}$ was toxic, and examination suggested acute appendicitis with peritonitis. At laparotomy, through a grid-iron incision, a perforated retrocaecal appendix was found with free pus in the peritoneal cavity. Culture of the pus subsequently yielded a profuse growth of Escherichia coli. After appendicectomy thorough peritoneal lavage with tetracycline in saline was carried out, and the wound was closed without drainage. No other antibiotic was given.

After the operation he had a slight fever, which was attributed to a low-grade wound infection. The sutures were removed on the sixth postoperative day, and on the same day he developed quite suddenly a painful, hot, red swelling of the right side of his scrotum. Torsion of the right testis was provisionally diagnosed and exploration carried out. On opening the tunica vaginalis $5 \mathrm{ml}$ of pus was released, but the testis and epididymis were normal. The wound was drained. His fever settled rapidly and he made an uneventful recovery. Culture of the pus proved sterile. Subsequent outpatient review at three months showed no evidence of further sepsis.

\section{Comment}

Co-existence of a perforated appendix and right-sided congenital hydrocoele or hernia must be common, yet we could find no report of residual sepsis in the scrotum. Our patient had no history or clinical finding suggestive of a hydrocoele or hernia, but presumably a connection between the peritoneal cavity and the tunica vaginalis was present. A patent processus vaginalis was not found at the second operation, but obliteration of the channel after the sepsis is probable. Spread of the sepsis from the abdominal wound into the inguinal canal seems unlikely, as the sepsis in the scrotum was confined within the tunica vaginalis.

Acute epididymo-orchitis after prostatic surgery is well recognised, infection reaching the testis via the vas deferens. It might be postulated that retroperitoneal infection from a retrocaecal appendix could track alongside the vas, but in this case there was no evidence of infection of the epididymis or testicle.

Opponents of intraoperative peritoneal lavage believe that it may spread sepsis within the peritoneal cavity. We have been unable to find any evidence for this view, but it is conceivable that the lavage encouraged the passage of organisms down a patent processus. It is unlikely that the use of systemic antibiotics would have prevented this occurrence. Recent (unpublished) observations have shown that tetracycline lavage leads to a therapeutic serum tetracycline concentration within an hour, and the pus trapped in the scrotum was shown to be sterile. It is therefore difficult to know how this complication might be avoided, even when a congenital hernia or hydrocoele is suspected before operation.

We thank Mr S S Miller, consultant surgeon at the Royal Aberdeen Children's Hospital, for permission to report on his patient.

${ }^{1}$ Campbell WB. Prophylaxis of infection after appendicectomy: a survey of current surgical practice. $\mathrm{Br} \mathrm{Med} \mathcal{F}$ 1980;281:1597-600.

2 Stewart DJ, Matheson NA. Peritoneal lavage in appendicular peritonitis. Br f Surg 1978;65:54-6.

${ }^{3}$ Stewart DJ. Antibiotic peritoneal lavage in the treatment of peritonitis. ChM thesis, University of Aberdeen, 1978.

4 Pinto DJ, Sanderson PJ. Rational use of antibiotic therapy after appendicectomy. Br Med f 1980;280:275-7.

(Accepted 16 February 1982)

Royal Aberdeen Children's Hospital, Aberdeen AB9 2ZD

W S MCKERROW, FRCS, surgical registrar

Aberdeen Royal Infirmary, Aberdeen AB9 2ZG

H J THOMSON, FRCS, surgical registrar (now at Royal Northern Infirmary, Inverness)

\section{Hypophosphataemic vitamin-D resistant rickets may need phosphate supplements}

The commonest form of vitamin-D resistant rickets is associated with a renal tubular defect producing hyperphosphaturia and hypophosphataemia, and has been treated with vitamin D up to 100000 units daily together with phosphate supplements. ${ }^{1}$ Recent reports ${ }^{2} 3$ suggest that phosphate supplements are not required when treatment is with $1 \alpha$-hydroxy vitamin $\mathrm{D}_{3}\left(1 \alpha-\mathrm{OHD}_{3}\right)$, but the results show that serum phosphate concentrations remained subnormal. We describe a child in whom rickets was not controlled with $1 \alpha$-hydroxy vitamin $\mathrm{D}_{3}$ until oral phosphate supplements were given.

\section{Case report}

A girl delivered at full term after breech presentation weighed $3150 \mathrm{~g}$ and was bottle fed. Welfare clinic multivitamin drops were given daily for one year. Her parents, brother, and sister were healthy and there was no family history of bone disease. She was very active, had a good appetite, and the milestones of development were normal. She walked at 13 months but her gait was waddling and her legs became increasingly bowed. When she presented at 23 months her height was -3 SD and weight -2 SD from the mean $(76 \mathrm{~cm}$ and $9.6 \mathrm{~kg}$ ). Radiographs showed evidence of severe rickets affecting wrists and knees. Results of investigations were: serum calcium concentration $2.5 \mathrm{mmol} / 1(10.1 \mathrm{mg} / 100 \mathrm{ml})$; phosphate concentration $0.7 \mathrm{mmol} / 1(2.1 \mathrm{mg} / 100 \mathrm{ml})$; alkaline phosphatase activity grossly raised; 